\title{
Dealing with inaccuracies in the analysis on solvent-induced swelling of transparent thin films using in situ spectroscopic ellipsometry in the visible wavelength range
}

Kristianne Tempelman, ${ }^{\text {a }}$ Emiel J. Kappert, ${ }^{\text {a,c }}$ Michiel J.T. Raaijmakers, ${ }^{\text {a }}$ Herbert Wormeester, ${ }^{\text {b }}$

Nieck E. Benes ${ }^{\mathrm{a}, *}$

${ }^{a}$ Films in Fluids, ${ }^{b}$ Physics of Interfaces and Nanomaterials, Department of Science and Technology, MESA ${ }^{+}$Institute for Nanotechnology, University of Twente, P.O. Box 217, 7500 AE Enschede, The Netherlands.

'Present address: BASF SE, D-67056 Ludwigshafen, Germany.

${ }^{*}$ Corresponding author: n.e.benes@utwente.nl

\section{Abstract}

The accuracy of spectroscopic ellipsometry studies of transparent polymer films in the visible wavelength range is greatly reduced in the presence of a liquid ambient. The relatively high refractive index of a liquid ambient strongly amplifies the effects of experimental non-idealities, such as window birefringence and an unknowingly inaccurate description of the optical properties of the ambient. In particular for thin films (approximately $<100 \mathrm{~nm}$ ), the implications of even small non-idealities are substantial, and large deviations in the thickness and refractive index are found. The concurrent low root mean square error (MSE) values show that a good MSE does not show that the optical model is correct, it just shows a good agreement between the experimental and fitted data. This implies that quantitative in situ ellipsometry studies for thin films are intricate, and derived properties like sorption behaviour should be treated with caution. Here, we show that quantitative in situ ellipsometry swelling measurements in solvents require a very accurate solvent dispersion relation, a high-quality temperature control, especially to prevent temperature differences between the cell, its content and exterior ambient, 
and accurate quantification of window effects, in particular for polymer films with thicknesses of approximately below $100 \mathrm{~nm}$, depending on the optical contrast of the polymer-solvent system.

Keywords: In situ spectroscopic ellipsometry, measurement accuracy, liquid ambient, thin polymer film swelling

\section{Introduction}

Applications of thin films are widespread. In many instances, these films are formed from, or can be exposed to, solvents or their vapours. In thin film production, solvents are for instance used during coating, ${ }^{[1]}$ in solvent annealing, ${ }^{[2]}$ or in characterizing a material by ellipsometric porosimetry ${ }^{[3]}$. Some films find their application in solvents, for instance as a sensor, ${ }^{[4,5]}$ or as a membrane. ${ }^{[6,7]}$ Typically, the structure of a thin film changes when it is exposed to a solvent. Tracking its structure in situ during exposure can provide crucial insights into the stability and properties of the film. The required accuracy for such measurements depends on whether one is only interested in trends or in accurate recorded values. Depending on the desired accuracy, a suitable technique can be chosen to track the in situ changing of the thin film structure. A diverse range of techniques is available for characterizing thin films in solvents, including spectroscopic ellipsometry, ${ }^{[8,9]}$ infrared/Raman spectroscopy, ${ }^{[10]}$ quartz crystal microbalance, ${ }^{[11]}$, white light interferometry ${ }^{[12]}, \mathrm{x}$-ray reflectivity ${ }^{[13]}$ and atomic force microscopy. ${ }^{[14]}$

Spectroscopic ellipsometry has proven to be a powerful technique for studying thin films in solvents, because it allows for determining both the thickness and the refractive index of the film, it is fast, and it can be applied both in situ and in operandi. ${ }^{[8]}$ When used correctly it can effectively capture features such as solvent gradients, ${ }^{[15]}$ anisotropy ${ }^{[16]}$ and solvent volume fractions ${ }^{[17]}$ that can develop and/or disappear from films upon solvent exposure. 
In addition to the wide variety of possible measurement configurations, modern spectroscopic ellipsometers are, in theory, sensitive to measure changes in layers with a resolution $\Delta d$ of 0.1 Å. ${ }^{[18]}$ For simple, transparent thin films on a well-defined substrate such as a silicon wafer, this sensitivity can be reached, as measurement accuracy is mainly limited by the precision of the ellipsometer in determining $\Psi$ and $\Delta$. For more complex samples, the choice of the optical model used to describe the film can have a strong impact on the accuracy. ${ }^{[18]}$ Here, precision is referred to as a random error, an inherent error which cannot be prevented and will always be there. Accuracy is a systematic error and is the result of the experimental design.

\begin{tabular}{|c|c|c|c|}
\hline Precise & Accurate & $\begin{array}{c}\text { Inaccurate } \\
\text { (systematic error) }\end{array}$ \\
\hline Imprecise & & \\
\hline
\end{tabular}

Figure 1. A schematic representation of either an accurate, precise or both an accurate and precise measurement. A precise measurement represents some tight grouping on a part of the dartboard, while an accurate measurement represents measurements that are in or close to the centre.

Deriving accurate information from in situ measurements, of a film exposed to a liquid solvent or vapour, is even more intricate than for dry systems. This is, among many other factors, due to the presence of a measurement cell (with windows), the presence of a solvent, temperature offsets, and temperature gradients within the measurement cell. Also the behaviour of the thin polymer film itself, such as the development of a gradient upon solvent exposure, is often 
unknown beforehand. Corresponding inaccuracies that are introduced by non-idealities that can be avoided are here referred to as systematic errors; when they are due to non-idealities that are difficult or impossible to avoid, we refer to them as random errors. The accuracy of in situ swelling experiments is in general not limited by the resolution of the apparatus itself, but by these additional random errors. These random errors will differ for each polymer-solvent system.

Although the accuracy of modern measurement equipment is widely discussed in the literature (see for instance, ${ }^{[19]},{ }^{[20]}$ or ${ }^{[21]}$ ), the topic of measurement accuracy within measurement cells has received far less attention. ${ }^{[22,23]}$ The loss of accuracy that one will experience during experiments involving measurement cells filled with a liquid solvent remains largely undocumented.

Information about the impact of common non-idealities is required to interpret the final data values. This requires careful judgement of which errors should be prevented during measurement, which parameters should be compensated for during modelling, and which parameters have to be accepted as random errors. During an ellipsometry measurement, the polarized light beam has to pass through the transparent windows of the measurement cell. These windows are often subjected to strain, which causes optical retardance of the light. The resulting change in the polarization state of the light results in an offset in the value of $\Delta$, one of the two angles that describe the ellipticity of the polarized light beam. ${ }^{[24]}$ For a well-designed cell filled with air the delta offset can be very low. For a cell that is filled with a liquid, the increased refractive index of the ambient strongly enhances the offset. ${ }^{[23,25-30]}$ To prevent a delta offset, a perfect alignment of the polarizer source to cell, and of the cell to the receiver unit is needed. This is only possible when the spectroscopic ellipsometer has the possibility to adjust the angle of the source and receiver unit separately, a luxurious ability only a few ellipsometry devices possess. The use of a solvent, also introduces systematic and random errors in the 
optical model. An accurate quantification of the swelling of a polymer film, induced by a solvent, requires very accurate information about the optical dispersion of the solvent. We learned that this is by no means trivial, as the literature reports many inconsistent data sets, resulting in the use of erroneous values for solvent dispersions in the optical model. In the case of the optical model, the solvent has two roles; as an ambient and as a dilutant in the polymer film. For this reason, changes in the optical dispersion, caused by variations in temperature or impurities, will affect the measured values of the thickness and the refractive index of the swollen film. When dealing with very thin films, the interaction with the solvent and the interface of the polymer will also have an effect on the optical dispersion of both the solvent and polymer. As a result, deriving the concentration of the solvent in the swollen film from an effective medium approach is also strongly affected. Finally, the use of a cell can affect the angle of incidence of the light beam. In particular, the effects of non-normal light incidence of the beam through the cell windows will cause an offset in the angle of incidence, which is amplified by an increased refractive index of the cell content. ${ }^{[30]}$ A slight tilt of the sample resulting from solvent flow will also affect the error in the angle of incidence.

The effect of a delta offset, the angle of incidence and the overall presence of a liquid inside a measurement cell, makes the design of such a measurement cell far from trivial. Also much care should be taken to limit the errors that can occur during in situ ellipsometry solvent swelling experiments. Table 1 gives an overview of possible random and systematic errors that are relevant for in situ ellipsometry solvent swelling experiments.

This paper discusses in detail the importance of uncertainties in in situ ellipsometry studies involving liquid solvents. The impact of uncertainties will strongly depend on the sample characteristics (e.g., the substrate, the film thickness, and the refractive indices of the solvent and the film). We selected a model system that is representative for an artificial membrane, consisting of a transparent film on a silicon substrate. The transparent film either has a low 
refractive index (reminiscent of polydimethylsiloxane, PDMS, $n=1.41$ ) or a high refractive index (reminiscent of a state-of-the-art polyimide, P84, $n=1.69$ ). ${ }^{[6,7]}$ The film thicknesses are varied in the range of 5 to $1000 \mathrm{~nm}$, to cover ultra-thin to micron-thick films. A silicon wafer is chosen as the substrate, because of its widespread use in this type of measurements. ${ }^{[8]}$ Silicon wafers can be considered as ideal substrates; they provide a smooth surface, well-known optical parameters, and a high refractive index. Water $(n=1.33)$ and toluene $(n=1.49)$ are selected as a low-and a high-refractive-index-solvent, respectively.

\section{Theory}

\section{$2.1 \Psi$ and $\Delta$}

During a spectroscopic ellipsometry measurement the change in the polarization state of the propagating light is measured. The change in polarization state is described by ratio of the reflectivities of linearly polarized light parallel to the plane of incidence $\left(\tilde{r}_{\mathrm{p}}\right)$ and perpendicular to the plane of incidence $\left(\tilde{r}_{\mathrm{s}}\right)$ :

$$
\rho=\tan (\Psi) \operatorname{cxp}(i \Delta)=\frac{\tilde{r}_{\mathrm{p}}}{\tilde{r}_{s}}
$$

where the $\Psi$ is the relative amplitude of the propagating $p$ and $s$ waves and $\Delta$ resembles the phase change along the propagation axis. ${ }^{[31]}$ The sign of $\Delta$ determines the sense of motion of the ellipse: $\Delta>0$ corresponds to a right-handed polarization and $\Delta<0$ to a left-handed polarization.

The $\Psi, \Delta$-spectra obtained in a spectroscopic ellipsometry experiment relate to the properties of the sample and the ambient in which it is situated. To illustrate this, Figure 2 shows the $\Psi, \Delta$ trajectories (generated with Woollam CompleteEase software) for polymer films in liquid water or toluene as ambient. For a high-index polymer, thicknesses changes affect both the $\Psi$ and $\Delta$ 
signals. For a low-index polymer the changes in $\Psi$ are significantly smaller than those observed for a high-index polymer. For low thicknesses $(<10 \mathrm{~nm})$, changes in $\Delta$ can be observed, but little or no changes in $\Psi$ are recorded. This difference in sensitivity towards signal changes of $\Psi$ and $\Delta$ is known as the thin film approximation ${ }^{[31]}$. Figure 3 illustrates the optical contrast between polymers and solvents. In the case of low optical contrast (e.g., a low-refractive-indexpolymer in a high-refractive-index-solvent), changes in $\Delta$ are more pronounced than changes in $\Psi$. Given sufficient optical contrast (high-refractive-index-polymer in low-refractive-indexsolvent), changes in $\Psi$ become more significant than in case of low optical contrast, and already at thicknesses higher than $5 \mathrm{~nm}$ both $\Psi$ and $\Delta$ changes will be observed.

A retardation of the light is the result of a generated phase difference between the $p$ - and $s$ polarizations $(\Delta) \cdot{ }^{[18]}$ Light retardation can be the result of density changes of the polymer film, but also windows of the measurement cell can introduce small retardations of the light, due to the presence of strain within these windows. In the supplementary, Tables 1 and 2 provide an indication of the delta-offset, a quantification of the window strain, for different measurement cells. It should be noted that the determination of a delta offset, especially in a liquid ambient is not always straightforward. In Figure 2, the simulated changes in $\Delta$, ranging from $-10^{\circ}$ to $10^{\circ}$, induced by the presence of strain in the windows, for a bare silicon wafer are represented by the red diamonds. Due to the window effects it appears as if a thin film is present, whereas the sample in reality does not comprise such a film. As a consequence, for measurements with a low optical contrast between the polymer film and the liquid ambient, different film thicknesses are difficult to distinguish from the presence of window effects. In this case higher inaccuracies in the results will be obtained. Also, in Figure 2 an identical behaviour of the low index polymer for both a toluene and water ambient is shown, indicating that the $\Psi$ and $\Delta$ behaviour is strongly dependent on the difference in the refractive index between the ambient and the polymer film. 

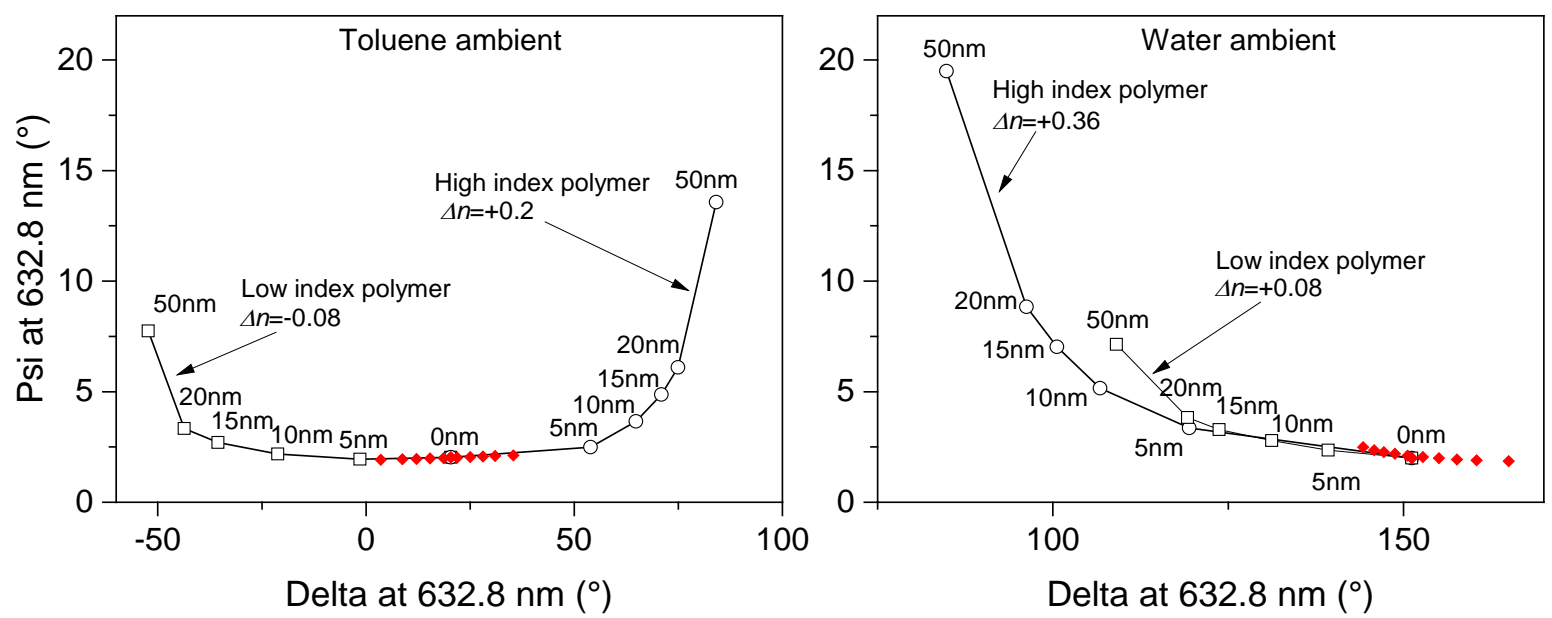

Figure 2. Simulated $\Psi, \Delta$-trajectories at $\lambda=632.8 \mathrm{~nm}$, of a high index polymer (squares) and a low index polymer (circles) in a toluene ambient (left panel) and in a water ambient (right panel) for various polymer film thicknesses $(0-50 \mathrm{~nm})$ on a silicon wafer. The red symbols represent the effect of an in-plane optical retardance by a window (simulated delta offsets in the range $-10^{\circ}$ to $10^{\circ}$ ) on $\Psi$ and $\Delta$ for a bare Si wafer.

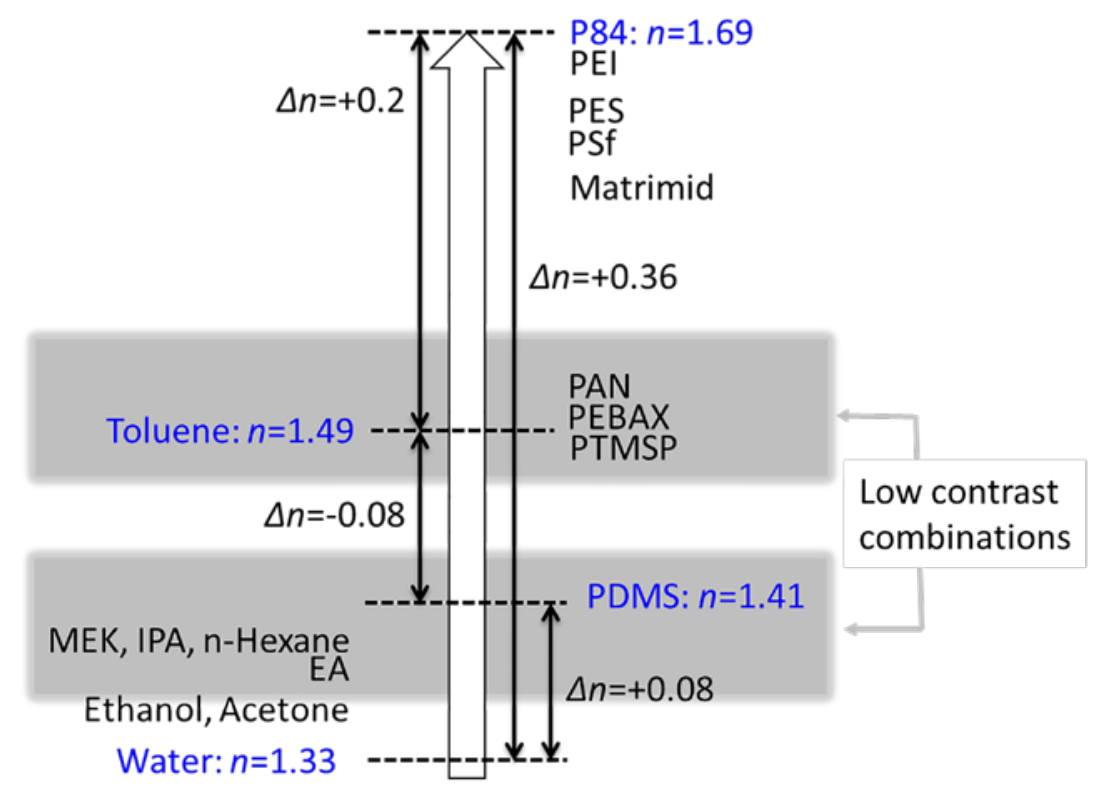

Figure 3. Ambient indices and polymer refractive indices. When the indices of the ambient and polymer are overlapping or are close to each other, as is displayed by the grey squares, there is a low optical contrast.

\subsection{Angle of incidence offsets}

The highest sensitivity for a spectroscopic ellipsometry measurement is obtained when the difference between $\tilde{r}_{\mathrm{p}}$ and $\tilde{r}_{\mathrm{s}}$ is maximized, because then the ratio of the amplitude $\left(\tilde{r}_{\mathrm{p}} / \tilde{r}_{\mathrm{s}}\right)$ is 
the largest. For this reason, an ellipsometric measurement is performed at the angle where the largest variation between $\Psi$ and $\Delta$ is to be seen. The highest amplitude ratio can be achieved when measurements are performed at an angle of incidence that is at about the Brewster angle $\left(\theta_{\mathrm{B}}\right)$, where $\tilde{r}_{\mathrm{p}}$ is equal or close to zero. ${ }^{[18]}$ When the sample is slightly tilted, or when the incoming light beam is not aligned exactly perpendicular to the windows, or when the polarizer and the analyser are not exactly aligned, an additional offset in the ratio between the two reflectivities is measured. Small angle changes will mainly result in an offset in $\Delta$, while larger changes will also cause an offset in $\Psi .{ }^{[31]}$ Therefore, the offsets in the angle of incidence should be kept to a minimum.

\section{Data Simulation}

\subsection{Representative base cases}

The impact of measurement uncertainties is assessed from data simulations for selected base case systems. All base case systems are based on a hypothetical sample that consists of a silicon wafer (with $2 \mathrm{~nm}$ native oxide layer), a thin homogeneous transparent film, and a transparent solvent as ambient. The differences between the base cases are the refractive indices of the film and the solvent. For each base case, $\Psi$ and $\Delta$ spectra were generated for an idealized system (no errors) using the Simulate Data option of the software package CompleteEase (J.A. Woollam Co.). Subsequently, the spectra were fitted using the same software, while introducing a certain deviation in the value of the systematic error of interest. For instance, the value of the offset in $\Delta$ is held $\neq 0$. Fitting parameters are the thickness of the polymer film, and the $A$ and $B$ constants of the Cauchy relation that describes the dispersion of this film. In all simulations and data fits a wavelength range of $370 \mathrm{~nm}$ - $1000 \mathrm{~nm}$ was used. Cauchy dispersions were used to generate a polymer film with a low refractive index (resembling PDMS) and a polymer film with a high 
refractive index (resembling P84). Thicknesses of 5, 20, 100, 200 and $1000 \mathrm{~nm}$ were selected to represent the relevant thickness range for thin film applications.

Water, using an optical dispersion fitted to the data published by Pribil and Synowicki, ${ }^{[32]}$ (H2O_high_purity_20_deg.mat, from the CompleteEase library), and toluene based on refractive index data published in ${ }^{[33]}$, were selected as solvents with low and high refractive indices, respectively. In Table 2 an overview of the Cauchy parameters of the materials used for the representative base cases is presented.

Three combinations of the refractive indices of the polymer and the ambient have been studied:

1. Low index polymer - high index solvent $\left(n_{\mathrm{p}}^{\text {kow }} n_{\mathrm{s}}^{\text {high }}\right)$;

2. High index polymer - low index solvent $\left(\boldsymbol{n}_{\mathrm{p}}^{\text {high }} n_{\mathrm{s}}^{\text {how }}\right)$;

3. High index polymer - high index solvent $\left(n_{\mathrm{p}}^{\text {high }} n_{\mathrm{s}}^{\text {high }}\right)$.

Two of these cases represent sufficient to high optical contrast between the polymer layer and the solvent (case $\mathbf{2}$ and 3). Case $\mathbf{1}$ represents a case with low optical contrast.

\subsection{Introducing non-idealities}

The simulation data of the base cases as described above, was generated using known ambient dispersions and without window offsets. To quantify non-ideality effects on the final thickness and refractive index, non-idealities were introduced during subsequent fitting of the modelled base cases. The obtained values for the root mean squared error (MSE) are shown as obtained. The MSE is a quantity that indicates the agreement between the optical model and the original experimental data. In the CompleteEase software, the MSE is defined as: ${ }^{[34]}$

$$
M S E=\sqrt{\frac{1}{3 \ell-m} \sum_{i=1}^{\ell}\left[\left(N_{\mathrm{E}_{i}}-N_{\mathrm{G}_{i}}\right)^{2}+\left(C_{\mathrm{E}_{i}}-C_{\mathrm{G}_{i}}\right)^{2}+\left(S_{\mathrm{E}_{i}}-S_{\mathrm{G}_{i}}\right)^{2}\right]} \times 1000
$$


where $\ell$ is the total number of wavelengths, $m$ the number of fit parameters, and $N=\cos (2 \Psi)$ , $C=\sin (2 \Psi) \cos (\Delta), S=\sin (2 \Psi) \sin (\Delta)$. Parameters subscripted with E represent the experimental (here: simulated) data while parameters subscripted with $G$ represent the fit data (that are fitted to the E data).

The changes in thickness and refractive index from the original thickness and from the refractive index are expressed by:

$\delta_{n}=\frac{n_{\text {fit }}-n_{0}}{n_{\text {fit }}} \times 100 \%$ and $\delta_{d}=\frac{d_{\text {fit }}-d_{0}}{d_{\text {fit }}} \times 100 \%$

where $\delta$ describes the deviation between the simulation thickness $\left(d_{\mathbf{0}}\right)$ or simulation refractive index $\left(\boldsymbol{n}_{\mathbf{0}}\right)$ and the fitted thickness $\left(d_{\mathrm{fft}}\right)$ or fitted refractive index $\left(\boldsymbol{n}_{\mathrm{fit}}\right)$.

\subsubsection{Effects of an inaccurate optical dispersion for the ambient}

Inaccuracies in the optical dispersion of the ambient were introduced as a constant offset or via a change in the shape of the dispersion. For toluene, the deviations in the optical dispersion were taken from literature. Here, the dispersion of Samoc, ${ }^{[33]}$ which is based on a meta-analysis of multiple studies, was considered the correct dispersion. The deviating dispersion given by Rubio ${ }^{[35]}$ was assumed to be inaccurate in this work. To create two other cases, two false dispersions were created by applying an offset of +0.005 and -0.005 in the Cauchy parameter A. These offsets correspond to a $\pm 8^{\circ} \mathrm{C}$ temperature deviation in the toluene ambient.

In Table 3 an overview of the Cauchy parameters of the deviating toluene optical dispersions are given.

For water, the dispersions available in the CompleteEase materials library, $\mathrm{H} 2 \mathrm{O}$ high purity 20degC.mat, $\mathrm{H}_{2} \mathrm{O}$.mat, $\mathrm{H}_{2} \mathrm{O}$ (Sellmeier).mat and $\mathrm{H}_{2} \mathrm{O}$ Pribil at $25^{\circ} \mathrm{C}$, were taken as the optical 
dispersions. Of these, $\mathrm{H} 2 \mathrm{O}$ high purity 20degC.mat is based on an extensive dataset and was considered the 'correct' dispersion. In Table 4 an overview of the Cauchy parameters of the deviating water optical dispersions are given.

\subsubsection{Swollen films}

A generated data set of 20, 40, 200 and $2000 \mathrm{~nm}$ films in toluene for which the refractive index was mapped by a Bruggeman Effective Medium Approximation (EMA) with 50\% solvent fraction, indicating the film contained of $50 \%$ toluene and $50 \%$ polymer materials, and was used to simulate swollen films in toluene with a swelling degree $(S D)$ of $100 \%$. The swelling degree is defined as:

$S D=\left(\frac{d_{\text {swollen }}}{d_{\text {dry }}}-1\right) \times 100 \%$

where $d_{\text {swollen }}$ is the thickness of the swollen polymer and $d_{\text {dry }}$ is the thickness of the dry polymer. The refractive index of the polymer film was held fixed, while both the thickness of the polymer film and the solvent volume fraction were fitted for the different Cauchy dispersions of the ambient (see 3.2.1) to resemble the situation of tracking the swelling of a polymer film with a known index.

The change in the solvent volume fraction with respect to the original solvent volume fraction is expressed by:

$$
\delta_{\Phi}=\frac{\Phi_{\text {fit }}-\Phi_{0}}{\Phi_{\text {fit }}} \times 100 \%
$$

where $\delta$ describes the deviation between the simulation volume fraction $\left(\Phi_{\mathbf{0}}\right)$ and the fitted volume fraction $\left(\Phi_{\text {fit }}\right)$.

\subsubsection{Delta offsets}


The influence of in-plane optical retardance introduced by the glass windows of a measurement cell was simulated by generating a data set without delta offset. Then the simulated data was fitted for the thickness, and the $A$ and $B$ parameters of the thin film while introducing a forced delta offset of $-10^{\circ}$ to $10^{\circ}$.

\section{Results and discussion}

\subsection{Inaccurate optical dispersion of the ambient}

A change in temperature or the presence of an impurity will cause a change in the optical dispersion of the ambient (the solvent), which will influence the oscillation pattern of $\Psi$ and $\Delta$. When the film becomes substantially swollen by the ambient, the optical contrast is generally lowered and the accuracy of model-derived parameters is further reduced, as will be shown in paragraph 4.1.2.

\subsubsection{Non-swollen films}

In this section, the films are modelled not to swell in the presence of the solvent. To approach representative systems as used in, for example, membrane applications or coatings, a high index polymer in a low index solvent (i.e., P84 in water) and a low index polymer in a high index solvent (i.e., PDMS in toluene) have been simulated. The first system represents high optical contrast, whereas the latter system represents low optical contrast.

In Figure 4, the $M S E$, the normalised thickness change $\left(\boldsymbol{\delta}_{\mathbf{d}}\right.$ ), and the normalised refractive index change $\left(\delta_{n}\right)$ are shown as a function of the film thickness, for several (incorrect) optical dispersions of the ambient. The left panel shows the case of high optical contrast (i.e., P84 in water) and the right panel shows the case of low optical contrast (i.e., PDMS in toluene). In the supplementary information, Figure 1, a third case is presented where the optical contrast is moderate (i.e., P84 in toluene). The curves assigned “original” present the results of the fitting, 
when using the solvent dispersion parameters identical to that used for creating the simulated data. In this case, an MSE close to one was obtained, implying a small inherent deviation between fitted and simulated data. The "original" curve represents the measurement of precision, while the other curves represent the accuracy you can obtain for a certain thickness. In general, the MSE increased upon introduction of a deviation in the optical dispersion of the ambient.

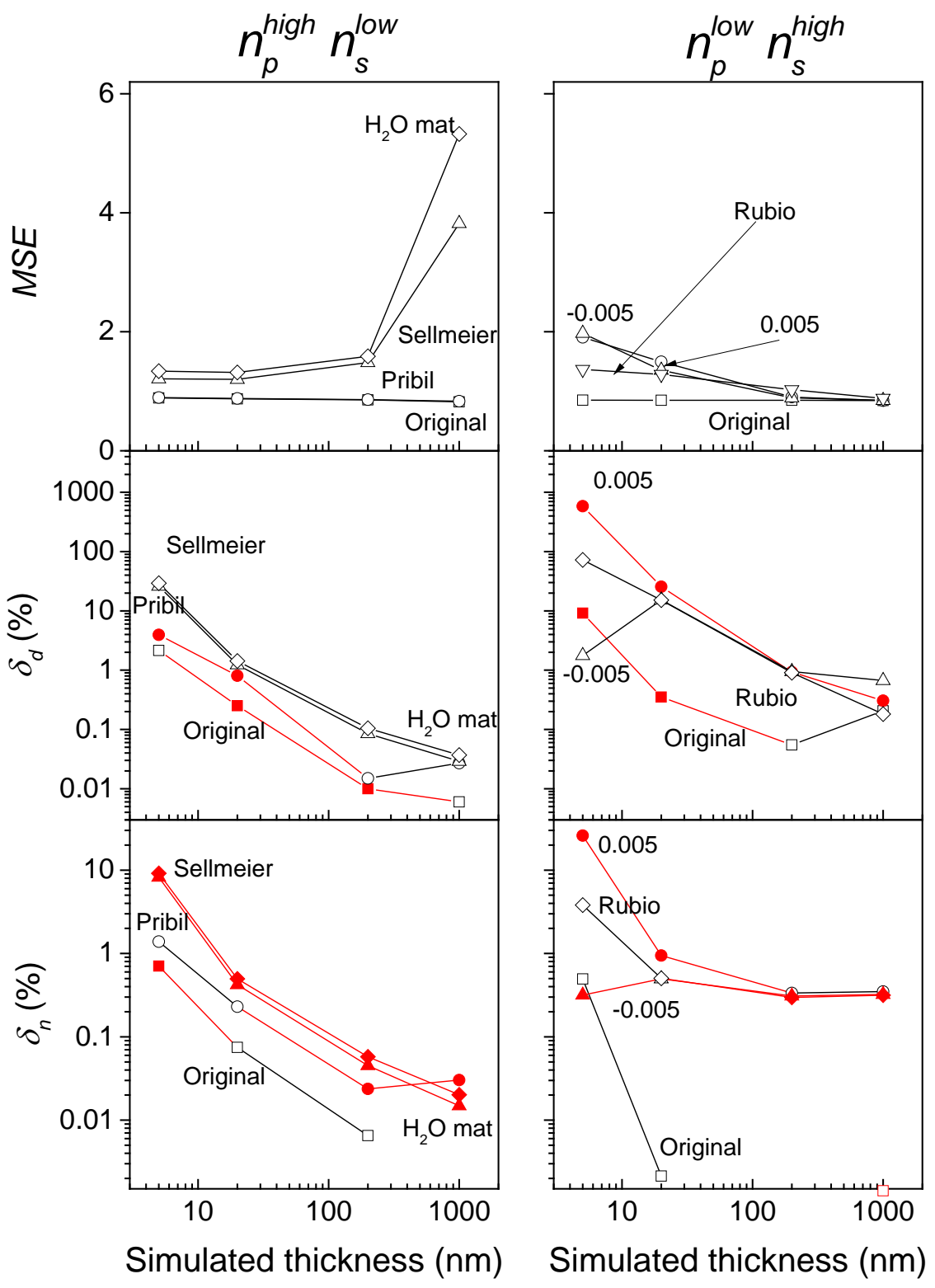

Figure 4. The MSE (upper row), $\delta_{d}$ (middle row) and $\delta_{n}$ (lower row) as a function of the simulated film thickness for various refractive index dispersions of a low-refractive-indexsolvent (left panel) and a high-refractive-index-solvent (right panel). Red symbols 
indicate negative values, i.e., an underestimation of the original values. The curve labelled original represents the inherent deviation that is obtained when using fitting parameters values identical to that used for creating the data. The other curves represent deviating optical dispersions.

In general a high correlation between the $A$ parameter and thickness was observed for all 5 and $20 \mathrm{~nm}$ films (ranging from -0.91 to 0.997) indicating that there is a relative high error margin for the thickness and refractive index obtained for 5 and $20 \mathrm{~nm}$ films compared to thicker films. This corresponds with the literature which described the correlation between the refractive index and the thickness for thin films. ${ }^{[31]}$

In the case of high optical contrast (Figure 4, left panel), an overall low MSE was obtained $(M S E<6)$ for a deviating optical dispersion of the ambient. The MSE remains low for the thinnest films and only shows a slight deviation $(<0.5)$ from the MSE obtained using the original index dispersion. With an increasing polymer film thickness, a higher MSE ( 5.3) for the deviating ambient optical dispersions was obtained. For $\delta_{d}$ and $\delta_{n}$, an opposite trend was visible; for a film of $5 \mathrm{~nm}, \delta_{d}$ up to $30 \%$ and $\delta_{n}$ up to $8 \%$ were observed, while for a film of $1000 \mathrm{~nm}$ only a $\delta_{d}$ and $\delta_{n}$ of $0.03 \%$ were observed. This can be explained by the fact that for thicker films, more polymer material will interfere with the light and thus the polymer contribution for the $\Psi$ and $\Delta$ signal increases. For thin films (approximately<100 nm), the amount of polymer that interferes with the light is lower, and the ambient will have a relative larger contribution to the light interference compared to thick films. In that case, the presence of the ambient is more pronounced and a change in the ambient will have more effect on the final results. The lack of $\Psi$ and $\Delta$ oscillations in these thin films, partly dampened by the larger amount of the ambient, causes the MSE to remain low. For the refractive index, a similar effect as that of the film thickness was observed. Also $\delta_{n}$ increases with decreasing simulated film thicknesses, but the changes in $\delta_{n}$ are one order of magnitude smaller as compared to $\delta_{d}$. The 
modelled thickness has a higher sensitivity to the changes in the ambient dispersions than the refractive index.

For low optical contrast cases (i.e., PDMS in toluene) (Figure 4, right panel), the MSE shows an opposite effect as observed for the case with high optical contrast. The MSE increases for decreasing film thicknesses and in general remains low $(<2)$. The generally low MSE can be explained by the fact that in case of low optical contrast, the $\Psi$ and $\Delta$ oscillations are strongly dampened, decreasing the sensitivity of the MSE to differences between the fit results and the experimental data. The trend observed in $\delta_{d}$ and $\delta_{n}$ is comparable to the case with high optical contrast, but the deviations observed were significantly higher. For $\delta_{d}$, deviations up to 585\% were observed for $5 \mathrm{~nm}$ and values up to $0.6 \%$ for $1000 \mathrm{~nm}$. For $\delta_{n}$, values up to $26 \%$ were observed for $5 \mathrm{~nm}$ and $0.3 \%$ for $1000 \mathrm{~nm}$. The observation of the high $\delta_{d}$ and $\delta_{n}$ values in combination with the low MSE for the case of low optical contrast, shows that the MSE can be misleading regarding the quality of the fit when working with thin films in an ambient (approximately $<100 \mathrm{~nm}$ ). In case of moderate optical contrast (See also Supplementray Information, Figure S1), the results for $\delta_{d}$ and $\delta_{n}$ are in between the values in case of high and low optical contrast. Also, the MSE in general lowers with a decrease in optical contrast. This lowering shows that a small change in the ambient dispersion can result in significant deviations of the final interpreted results. It is therefore important to carefully control the environment of the experiments to cancel out any undesired ambient effects caused by external features such as temperature changes. Also, it is very important to obtain an accurate description of the ambient index dispersion. The literature does raise awareness on the complications that arise for the simultaneous determination of the optical constants and thickness of thin films (approximately<100 nm), ${ }^{[18,19,36]}$ but the effects of deviating ambient dispersions also have dramatic consequences on the final data interpretation. 


\subsubsection{Swollen films}

In the previous sub-section, no swelling of the polymer was assumed. However, when a polymer is exposed to a solvent such as toluene, the solvent will penetrate into the polymer and the polymer will start to swell to a certain extent. In this case, a change in the ambient causing a deviation in the refractive index dispersion will not only have an effect on the ambient simulation itself, but also on the refractive index of the swollen polymer.

In Figure 5, the MSE, $\delta_{d}$ and $\delta_{\Phi}$ are shown as a function of the film thickness, for several (incorrect) optical dispersions of the ambient. The left panel shows the case of high optical contrast (i.e., P84 in toluene) and the right panel shows the case of low optical contrast (i.e., PDMS in toluene).

The MSE of the fits of the swollen films, in the case of both sufficient and low optical contrast, shows a similar behaviour as observed for the non-swollen films in a liquid ambient with high optical contrast. For both moderate and low optical contrast, the MSE increases with increasing film thicknesses to MSE 6, when a deviating optical dispersion of the ambient was introduced.

In case of moderate optical contrast (i.e., P84 in toluene) (Figure 5, left), $\boldsymbol{\delta}_{\boldsymbol{d}}$ is $85 \%$ for 10 nm swollen films and $0.15 \%$ for $2000 \mathrm{~nm}$ swollen films. This is lower than for the non-swollen film. For $\delta_{\Phi}$, large deviations were observed. For very thin swollen films (i.e., a $10 \mathrm{~nm}$ swollen film), $\delta_{\Phi}$ shows that no solvent was detected in the polymer film (i.e., $\Phi=0$ ), resulting in an infinite $\delta_{\Phi}$. The dependency on the optical dispersion of the ambient by the solvent volume fraction can be explained by the dependency of the solvent volume fraction on the refractive index of the solvent and the refractive index of the polymer. The polymer refractive index was assumed to be constant for all cases, therefore every change in the refractive index of the solvent 
will largely influence the solvent volume fraction in the polymer. An alternative to obtaining the solvent volume fraction based on the refractive index, is by means of dilation based on the thickness data:

$$
\Phi_{\mathrm{s}}=1-\frac{d_{\mathrm{dry}}}{d_{\text {swollen }}}
$$

$\Phi_{\mathrm{s}}$ is the solvent volume fraction, $\boldsymbol{d}_{\text {dry }}$ the dry thickness of the polymer and $\boldsymbol{d}_{\text {swollen }}$ the equilibrated thickness of the swollen polymer. The dilation approach assumes that no excess free volume is present within the thin polymer film, there is no change in molar volume of the polymer, and solvent-induced volume changes are forced to occur only in the direction perpendicular to the substrate. Although in practice a calculation on the solvent volume fraction based on the dilation approach is often less reliable due to the presence of free volume and the confinement of the swelling direction, it allows for a rough estimate. In the case of the simulation results in this paper, the solvent volume fraction calculated based on the dilation approach should coincide with the solvent volume fraction based on the Bruggeman effective medium approximation, as no excess free volume is incorporated in the model. For films thicker than approximately $100 \mathrm{~nm}$ the deviation from the actual data is a factor ten lower using the dilation approach (see also Supplementary info, Figure S2). For thinner films, the use of EMA is more reliable. In case of very thin films (approximately lower than 20nm) both the EMA method and dilation method become very unreliable.

In the case of low optical contrast (i.e., PDMS in toluene) (Figure 5, right), for both $\delta_{\mathbf{d}}$ and $\delta_{\Phi}$ values comparable to the case of moderate contrast were obtained. To conclude, the inaccuracies in the refractive index and/or solvent volume fraction increase for swollen polymer systems compared to non-swollen polymer systems, while the inaccuracies in the thickness decrease compared to non-swollen systems but remain overall high. 

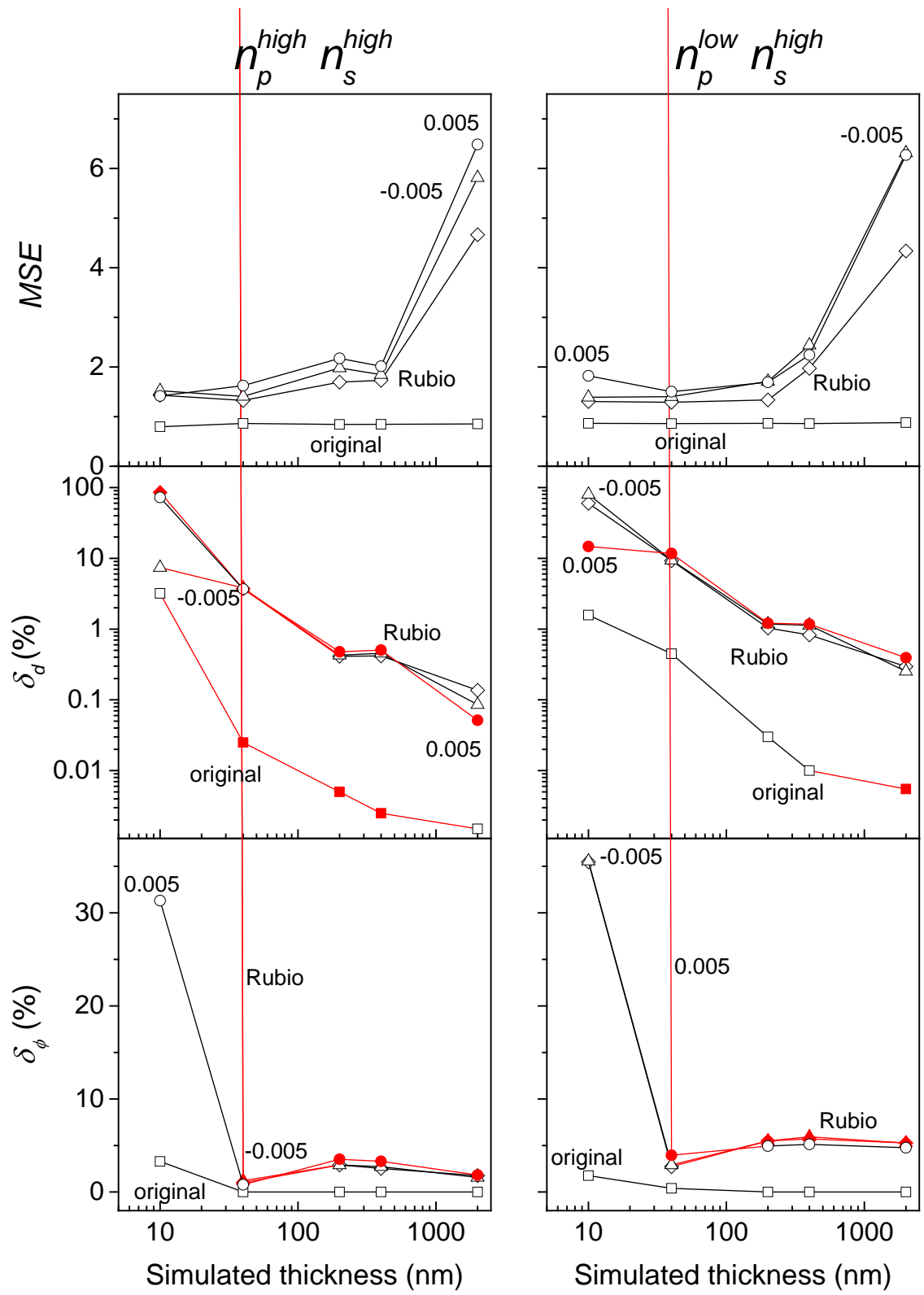

Figure 5. Simulation results for swollen films in a solvent. The MSE (upper row), $\delta_{d}$ (middle row), $\delta_{\Phi}$ (lower row) as a function of the simulated film thickness for various refractive index dispersions of a high-index solvent. Red symbols indicate negative values, i.e., an underestimation of the original values. The curve labelled original represents the inherent deviation that is obtained when using fitting parameters values identical to that used for creating the data. 


\subsection{Cell window effects in a liquid ambient}

In Figure 6 the MSE, $\boldsymbol{\delta}_{\boldsymbol{d}}$ and $\boldsymbol{\delta}_{n}$ are shown as a function of an induced delta offset in the optical model, as described in section 3.2.3. The left panel shows the case of high optical contrast (i.e., P84 in water) and the right panel shows the case of low optical contrast (i.e., PDMS in toluene).

Small disturbances in the optical light path, such as stresses in the optical windows, will result in a large change of the measured $\Psi$ and $\Delta$ signal. The effects will be more pronounced for thin films in comparison with thick films. For a disturbance in the optical light path to be detected in the measured signal of thick films, the disturbance must be significantly larger than for thin films as for thick films, the higher amount of polymer material will have a larger contribution to the light interference compared to the surrounding. In the case of thick films, small optical retardances of the light will give an offset in the $\Delta$ curve, contributing to an increase in the MSE. In general a decrease in the optical contrast dampens the $\Psi$ and $\Delta$ oscillations, resulting in a decrease of the MSE. This is shown in Figure 6 where the MSE of a $100 \mathrm{~nm}$ film strongly increases with increasing delta offsets for a case with high optical contrast, but overall remains low for a case with low optical contrast.

For $\boldsymbol{\delta}_{\boldsymbol{d}}$ (Figure 6, middle row), it can be observed that in the case of high optical contrast, the modelled thickness is an underestimation of the original simulated film thickness for positive delta shifts. The consequence of a delta shift is that the measured delta signal has an offset, shifting the $\Delta$ signal upwards (positive delta shift) or downwards (negative delta shift).

Also, $\boldsymbol{\delta}_{\boldsymbol{d}}$ increases with decreasing simulated film thicknesses, a behaviour similar to the trends witnessed in Figures 4 and 5 . The highest increases were observed for the first degree $\left(-1^{\circ}\right.$ and $1^{\circ}$ delta shift) in the delta shift; after this, the deviations increase gradually. This indicates that not correcting for a small delta offset (which can be due to additional strain in the windows due to O-ring swelling), will result in a large deviation for the modelled thickness of thin films 
(Approximately $<100 \mathrm{~nm}$ ). For the case in which there is low optical contrast, this effect is even more pronounced and also occurs for thick films. Figure 6 shows that even for a simulated $1000 \mathrm{~nm}$ film, a $\boldsymbol{\delta}_{\boldsymbol{d}}$ of $3 \%$ for a delta change of only $1^{\circ}$ was observed.

For $\delta_{n}$, in case of high optical contrast, an underestimation of the simulated refractive index was observed at a negative delta shift. This is presumably a compensation effect of the overestimated thickness at this point, and in order to maintain the same optical density, the refractive index is underestimated. In the case of low optical contrast (Figure 6, right panel), $\delta_{n}$ for a $1000 \mathrm{~nm}$ and $100 \mathrm{~nm}$ film is an order of magnitude higher compared to the cases with high optical contrast. This indicates that in case of low optical contrast, no accurate measurements can be performed for films thinner than approximately $100 \mathrm{~nm}$. This is in contradiction with the suggestion of a good fit by the low MSE $(<4)$ for $100 \mathrm{~nm}$ or thinner films.

The values for the delta shifts that have been used in this study, 1-5, are quite realistic for the rather aggressive solvents, such as the toluene considered here. Typical data for window strain effects of different kind of cells are listed in the Supporting Information. The data imply that, especially at elevated temperatures, delta offsets of our homebuilt cells can be high. This is due to several reasons, such as interaction between the solvent and the glue used to seal the cell, the temperature gradients from the outside to the inside of the cell, the combination of materials with distinct thermal expansion coefficients (e.g., glass body and quartz windows), and the large volumes ( 100 mL). The large volumes are chosen to facilitate fast insertion of samples into the liquid at $t=0 \mathrm{~s}$. The volume of modern commercial liquid cells is much smaller and is completely filled with the solvent. This reduces the existence of temperature gradients within the liquid itself. Also, the construction of commercial cell bodies out of stainless steel negates thermally induced stresses at the working temperatures of maximum $50^{\circ} \mathrm{C}$. The windows of the 
commercial cells are relative small and sealed with O-rings. This sealing makes them sensitive to strain when exposed to an aggressive solvent. In this study kalrez O-rings were used and still delta offsets increased upon solvent exposure. This indicates that the importance of sealants should be carefully considered.

The delta-offsets in the range of 5-10 are an extreme case and imply that one has not carefully considered the liquid cell design. As shown in Figure 6, the consequence of a badly designed cell on the final obtained thickness and optical constants is large.
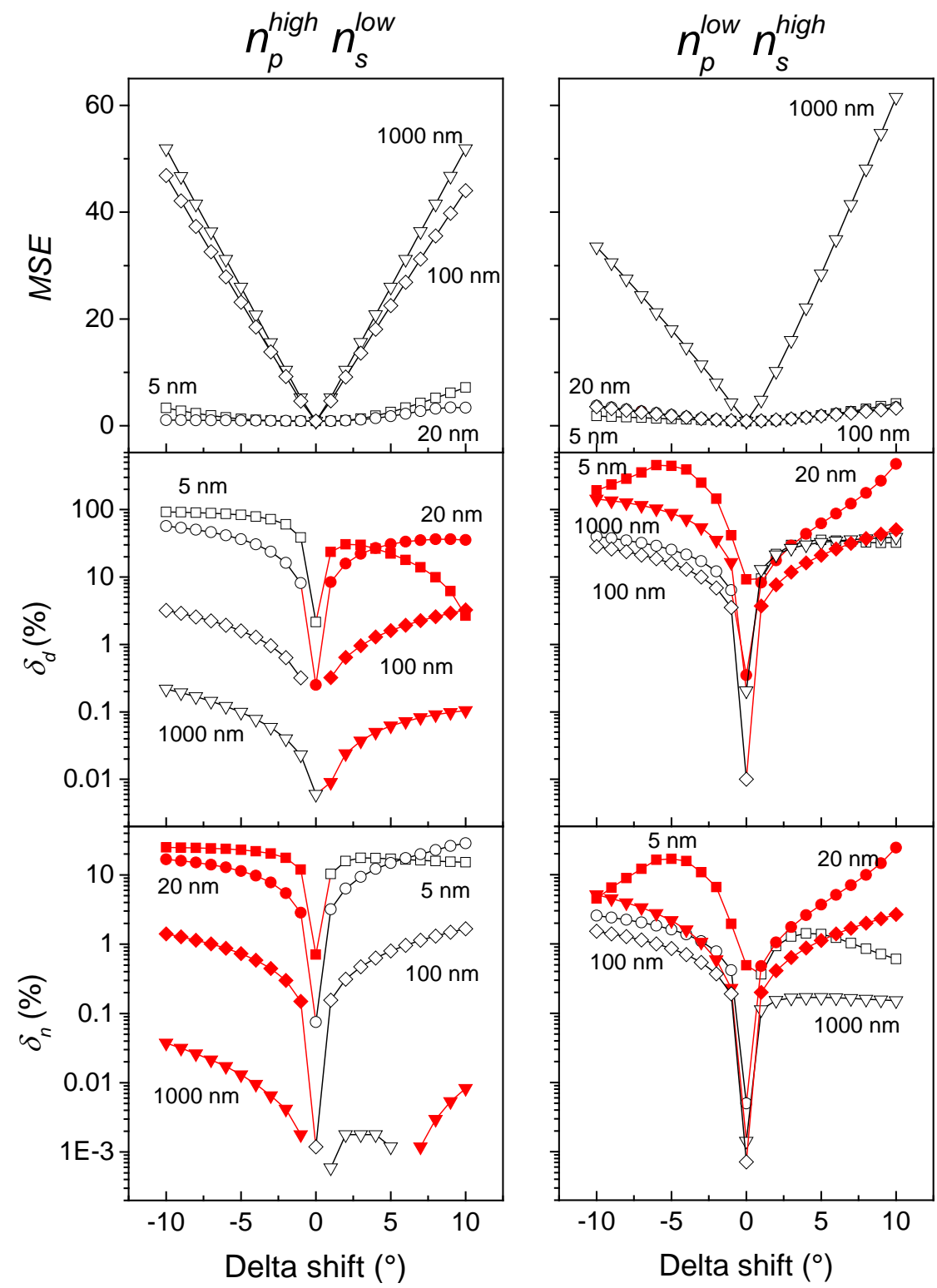
Figure 6. The MSE (upper row), $\delta_{d}$ (middle row), and $\delta_{n}$ (lower row) as a function of the delta shift $\left({ }^{\circ}\right)$ for a case of high optical contrast (left column) and low optical contrast (right column). Red symbols indicate negative values, i.e., an underestimation of the original values. The absence of a symbol in the deviation from the refractive index graphs indicate that the deviation is smaller than $10^{-3}$.

\subsection{General considerations}

For all the results shown in the previous sections, polymer films were considered that are ideal in the sense that surface roughness and solvent gradients are considered neglectable. For rubbery polymers like PDMS, which swell instantaneously when exposed to a solvent, this might be the case. However, for glassy polymers where swelling of the polymer film will be time dependent, the parameters such as surface roughness and gradients within the polymer film will change. This requires that these parameters must be included in the analysis and have a certain correlation with the index and thickness. Although more parameters may improve the match between model and the experimental data, the correspondence between the fitted and the actual values may decrease. Thus after adding a fit parameter, the fitted values should be treated with even more caution.

\section{Conclusions}

In this study, we discuss the reduced accuracy of in situ spectroscopic ellipsometry studies of polymer films, due to the exposure to a liquid ambient. It is shown that the relatively high refractive index of the liquid ambient strongly amplifies the effects of experimental nonidealities, such as window birefringence and an inaccurate optical dispersion relation for the liquid ambient. The effects of even small non-idealities are in particular large for thin films (Approximately $<100 \mathrm{~nm}$ ). For such thin films, large deviations in the thickness and refractive index are found, while the concurrent low MSE values erroneously suggest a good fit of the optical model to experimental data. This implies that a low MSE will not necessarily indicate an improved fit result, and that quantitative in situ ellipsometry studies for these thin films 
should be treated with caution. For films that do not substantially swell, the value of the thickness of the films is more sensitive to non-idealities than the refractive index. For films that do show substantial uptake of the liquid there is a very pronounced effect on the value of the solvent uptake, calculated from an effective medium approximation approach. For thin films, this value should be considered inherently inaccurate.

Our results demonstrate that quantitative in situ ellipsometry polymer swelling measurements in solvents require a very accurate solvent dispersion relation, a high-quality temperature control, especially to prevent temperature differences between the cell, its content and exterior ambient, and accurate quantification of window effects, in particular for polymer films of thicknesses up to $100 \mathrm{~nm}$. Multi-wavelength refractometry experiments on solvent (mixtures) and extensive window calibrations are crucial for quantitative in situ ellipsometry solvent swelling experiments. Although the quantitative results shown in this paper cannot be generalised for each swelling system used for spectroscopic ellipsometry, it shows that the accuracy is strongly dependent on the swelling system and it is vital to perform an accuracy analysis as shown above. 


\section{Acknowledgements}

This work is part of the research programme TA-ISPT Fundamentals with project number 731.014.203, which is (partly) financed by the Netherlands Organisation for Scientific Research (NWO).

\section{References}

[1] D.B. Hall, P. Underhill and J.M. Torkelson, Polym. Eng. Sci., 1998, 38,12.

[2] K.A. Cavicchi, K.J. Berthiaume and T.P. Russell, Polymer (Guildf)., 2005, 46, 25.

[3] M.R. Baklanov, K.P. Mogilnikov, V.G. Polovinkin and F.N. Dultsev, J. Vac. Sci. Technol. B Microelectron. Nanom. Struct., 2000, 18,3.

[4] Y. Sakai, Y. Sadaoka and M. Matsuguchi, Sensors Actuators B Chem., 1996, 35,1-3.

[5] I. Tokarev and S. Minko, Soft Matter, 2009, 5,3.

[6] P. Marchetti, M.F. Jimenez Solomon, G. Szekely and A.G. Livingston, Chem. Rev., 2014, 114.

[7] P. Vandezande, L.E.M. Gevers and I.F.J. Vankelecom, Chem. Soc. Rev., 2008, 37,2.

[8] W. Ogieglo, et al., Prog. Polym. Sci., 2015, 42.

[9] J.L. Keddie, Curr. Opin. Colloid Interface Sci., 2001, 6,2.

[10] P. Innocenzi, et al., J. Phys. Chem. A, 2008, 112,29.

[11] K.A. Marx, Biomacromolecules, 2003, 4,5.

[12] D. Magerl, et al., Macromolecules, 2015, 48,9.

[13] M. Mukherjee, M. Souheib Chebil, N. Delorme and A. Gibaud, Polymer (Guildf)., 2013, 54,17 .

[14] A.J. Parnell, et al., Soft Matter, 2009, 5,2.

[15] W. Ogieglo, H. Wormeester, M. Wessling and N.E. Benes, Polymer (Guildf)., 2013, 54,1 .

[16] R. Buchhold, et al., Thin Solid Films, 1999, 350,1-2.

[17] S.M. Sirard, P.F. Green and K.P. Johnston, J. Phys. Chem. B, 2001, 105.

[18] H. Fujiwara, Spectroscopic Ellipsometry: Principles and Applications. John Wiley \&Sons, Ltd, 2007.

[19] H. Arwin and D.E. Aspnes, Thin Solid Films, 1984, 113,2.

[20] K. Vedam, Thin Solid Films, 1998, 313-314.

[21] D.E. Aspnes, Thin Solid Films, 2004, 455-456.

[22] J.S. Papanu, D.W. Hess, A.T. Bell and D.S. Soane, J. Electrochem. Soc. , 1989, 136,4.

[23] R.M.A. Azzam and N.M. Bashara, J. Opt. Soc. Am., 1971, 61,5.

[24] B.J. Stagg and T.T. Charalampopoulos, J. Phys. D. Appl. Phys., 1999, 26. 
[25] L. Jin and E. Kondoh, Opt. Lett., 2014, 39,6.

[26] A. Straaijer, L.J. Hanekamp and G.A. Bootsma, Surf. Sci., 1980, 96.

[27] G.E. Jellison, Appl. Opt., 1999, 38,22.

[28] C.M. Herzinger and B.D. Johs, 2000.

[29] J.M.M. de Nijs and a. van Silfhout, J. Opt. Soc. Am. A, 1988, 5,6.

[30] J.S. Papanu, D.W. Hess, A.T. Bell and D.S. Soane, J. Electrochem. Soc., 1989, 136,4.

[31] H.G. Tompkins and E.A. Irene, Handbook of Ellipsometry. William Andrew, Inc, 2005.

[32] R.A. Synowicki, et al., J. Vac. Sci. Technol. B Microelectron. Nanom. Struct., 2004, 22,2004.

[33] A. Samoc, J. Appl. Phys., 2003, 94,9.

[34] CompleteEase ${ }^{\circledR}$ Software Manual. J.A. Woollam Co., Inc., 2011.

[35] J.E.F. Rubio, et al., Exp. Therm. Fluid Sci., 2004, 28,8.

[36] F.L. McCrackin, J. Opt. Soc. Am., 1970, 60,1.

[37] M.R. Querry, D.M. Wieliczka and D.J. Segelstein, Handbook of Optical Constants of Solids. Handb. Opt. Constants Solids, Elsevier, 1997. 
Table 1. An overview on the type of errors/uncertainties that can occur during a measurement and their effects on the final results.

\begin{tabular}{|c|c|c|}
\hline Source of error & Influence & Effect \\
\hline $\begin{array}{l}\text { Glass windows in } \\
\text { measurement cell }\end{array}$ & $\begin{array}{l}\text { Optical in-plane light } \\
\text { retardance, resulting in } \\
\text { delta offset }\end{array}$ & $\begin{array}{l}\text { In air or gasses (low } n \text { ): small } \\
\text { effect. } \\
\text { Solvents and compressed gasses } \\
\text { (higher } n \text { ): the effect can be } \\
\text { significant. }\end{array}$ \\
\hline $\begin{array}{ll}\text { - } & \text { Errors in optical } \\
\text { dispersion solvent: } \\
\text { Unknown dispersion } \\
\text { - } & \text { Incorrect dispersion } \\
\text { - } & \text { Impure solvent } \\
\text { - } & \text { Temperature deviations } \\
\text { - } & \text { Interaction solvent with } \\
& \text { polymer }\end{array}$ & $\begin{array}{l}\text { Erroneous dampening } \\
\text { effect in the optical model } \\
\text { on the } \Psi \text { and } \Delta \text { values. } \\
\text { Error in solvent } \\
\text { concentrations determined } \\
\text { from effective medium } \\
\text { approaches. }\end{array}$ & $\begin{array}{l}\text { Particularly significant for films } \\
\text { smaller than } \sim 100 \mathrm{~nm} \text { (vide infra) }\end{array}$ \\
\hline $\begin{array}{l}\text { Offset in the angle of } \\
\text { incidence } \\
\text { - } \quad \text { Angle offset of full cell } \\
\text { - } \quad \text { Angle offset of cell } \\
\text { bottom compared to } \\
\text { windows } \\
\text { - } \quad \begin{array}{l}\text { Non-normal incidence } \\
\text { in cell windows }\end{array}\end{array}$ & $\begin{array}{l}\text { Misalignment of the } \\
\text { polarizer and analyser, } \\
\text { resulting in offsets of the } \\
\Psi \text { and } \Delta \text { values. }\end{array}$ & $\begin{array}{l}\text { Apparent in all cases and should } \\
\text { be avoided. Effect is amplified } \\
\text { by higher value of the refractive } \\
\text { index of the cell content. }\end{array}$ \\
\hline
\end{tabular}


Table 2. An overview of the Cauchy parameters of the materials used for the simulation of the representative base cases.

\begin{tabular}{|c|c|c|c|c|}
\hline & $\bar{A}$ & $\bar{B}$ & $\bar{C}$ & $\boldsymbol{k}$ \\
\hline Low-refractive-index-polymer & 1.397 & 0.0004 & 0 & 0 \\
\hline $\begin{array}{r}\text { High-refractive-index- } \\
\text { polymer }\end{array}$ & 1.63 & 0.022 & 0 & 0 \\
\hline H2O high purity 20 degC* & 1.323 & 0.00316 & $7.6761 \cdot 10^{-6}$ & 0 \\
\hline Toluene (Samoc)** & 1.475 & 0.00699 & 0.000218 & 0 \\
\hline
\end{tabular}

*A Cauchy relation fitted to the optical dispersion data in ${ }^{[32]}$.

**A Cauchy relation fitted to the optical dispersion data in ${ }^{[33]}$. 
Table 3. An overview of the Cauchy parameters of the deviating optical dispersions for toluene.

\begin{tabular}{|r|c|c|c|c|}
\hline & A & B & C \\
\hline Toluene (Rubio)* & 1.471 & 0.00656 & 0.000258 & 0 \\
& & & & 0 \\
\hline Toluene (Offset +0.005) & 1.480 & 0.00699 & 0.000218 & 0 \\
\hline Toluene (Offset -0.005) & 1.470 & 0.00699 & 0.000218 & 0 \\
\hline
\end{tabular}

* A Cauchy relation fitted to the optical dispersion data in ${ }^{[35]}$. 
Table 4. An overview of the Cauchy parameters of the deviating optical dispersions for water.

\begin{tabular}{|r|c|c|c|c|}
\hline \multicolumn{2}{|c}{ A } & B & C \\
\hline $\mathrm{H}_{2} \mathrm{O}$ (Sellmeier)* & 1.319 & 0.00502 & $-1.5129 \cdot 10^{-5}$ & 0 \\
& & & & \\
\hline $\mathrm{H}_{2} \mathrm{O}$ (Pribil 25) & 1.323 & 0.00316 & $7.6760 \cdot 10^{-6}$ & 0 \\
& & & & 0 \\
\hline $\mathrm{H}_{2} \mathrm{O}^{* *}$ & 1.319 & 0.00518 & $-3.1206 \cdot 10^{-5}$ & 0 \\
& & & & \\
\hline
\end{tabular}

* A Cauchy relation based on a Sellmeier fit of data from ${ }^{[37]}$.

**Best fit by using Cauchy relation. Based on data from ${ }^{[37]}$. 